\title{
An Experimental Study on Superplastic Hemispherical Forming of Aluminum Lithium 2195 Alloy
}

\author{
Ho-Sung Lee, Jong-Hoon Yoon, and Joon-Tae Yoo
}

\begin{abstract}
Superplastic forming (SPF) technology is currently being used to produce a number of components for aerospace vehicles. The most important advantages of using SPF technology is the process simplicity and economy in tooling. The widely used superplastic materials are titanium alloys and aluminum alloys, which are the workhorse alloys of the aerospace industry. The low density and good mechanical properties of Aluminum-Lithium 2195 alloy make them successfully used in cryogenic tank of space vehicles. In this study, a hemispherical dome is produced with this alloy by superplastic forming. By controlling hydraulic gas pressure, it was possible to maintain the superplastic condition during the forming process. The forming temperature was $460^{\circ} \mathrm{C}$ to form a complete hemispherical dome with diameter of $350 \mathrm{~mm}$ for 3,420 sec. The programmed gas pressure was applied and the maximum pressure was limited to $5 \mathrm{MPa}$ to protect the forming tool. The distribution of thickness within the hemisphere was investigated and confirmed the analysis result. The process development for the production of a hemisphere with 2195 aluminum alloy has been achieved. The hemispherical dome is in good conformity with drawing dimensional and tolerance specifications.
\end{abstract}

Index Terms - Aluminum lithium alloy, gas pressure forming, hemisphere, superplastic forming.

\section{INTRODUCTION}

Superplasticity is the ability of fine-grained materials to exhibit extensive neck-free elongations during deformation at elevated temperatures which are above or at 0.5 of the melting point in Kelvin. Since it is a diffusion-controlled process at elevated temperatures, the thermally activated component of the dimensionless flow stress has been represented in a form of the following equation (Mukherjee-Bird-Dorn equation) [1], [2]:

$$
\frac{\dot{\varepsilon} k T}{G b D}=A\left[\frac{\sigma}{G}\right]^{n}\left[\frac{b}{d}\right]^{p}
$$

with

$$
D=D_{o} \exp \left\lfloor-\frac{Q}{R T}\right\rfloor
$$

where $\sigma$ is the flow stress, $G$ is the shear modulus, $b$ is the Burgers vector, $\mathrm{d}$ is the grain size, $n$ is the stress exponent, $p$ is the strain rate dependence on grain size, $k$ is the Boltzman constant, $\dot{\varepsilon}$ is the strain rate, $T$ is the absolute temperature, $A$ is

Manuscript received August 25, 2016; revised January 1, 2017. This work was supported in part by the Korean Ministry of Science, ICT and Future Planning (MSIP) through KSLV-II Program.

The authors are with the Korea Aerospace Research Institute, Daejeon, 34133, Korea (e-mail: hslee@kari.re.kr, yjh@kari.re.kr, ytyoo@kari.re.kr). a microstructure and mechanism dependent constant which varies with the controlling mechanism, $D$ is the appropriate diffusivity, $Q$ is the activation energy for the diffusion process that controls the deformation mechanism, and $D_{\mathrm{o}}$ is the frequency factor for diffusion [1]. For practical application of material parameters for superplastic forming, the strain rate and the stress exponent are important and should be monitored during the forming process. SPF technology provides superior formability and more saving of materials in final products than conventional forming processes do. Since the assembly includes only clamping dies, temperature chamber and regulated gas pressure to provide forming force, the system is easy to use. This method is especially advantageous when sheet metals are formed into complex shapes [3]. Since only single tool surface is necessary, tooling design of various different configurations can be easily modified and manufacturing lead time is short. The finished products have exceptional accuracy in dimension, good surface finish and isotropic properties.

Aluminum alloys usually do not exhibit superplastic properties under conventional processing conditions. However, some aluminum-lithium alloys like AA2090, AA8090, and AA2195 contain a relatively large amount of zirconium which provides a dispersion of very fine $\mathrm{Al}_{3} \mathrm{Zr}$ particles that stabilize the microstructure developed during rolling and prevent recrystallization during superplastic forming process [4]. This is because one of the requirements for superplasticity is to maintain the fine grain size at deformation temperature. Conventionally a relationship between grain size and flow stress is known to show lower flow stresses at larger grain sizes. However in superplastic forming process, strain hardening is caused by grain growth, because the grain boundaries are weaker than the grains themselves. $\mathrm{Al}_{3} \mathrm{Zr}$ particles inhibit subsequent recrystallization by pinning mobile grain boundaries at elevated temperatures.

The present work is to superplastically form a hemispherical dome with AA2195 using hydrostatic gas pressure. The optimum forming condition was obtained from biaxial bulging test [5]. During the pressurized bulging test, the height of the bulged sheet is measured and material parameters of flow stress are determined in bi-axial stress state based on the membrane theory. The experimental result is compared with the analytical result.

\section{EXPERIMENTAL}

The material was received from Constellium Aerospace, France as sheets of $1,500 \times 1,350 \times 7.62 \mathrm{~mm}$. Finite element simulation has been carried out using commercial finite 
element code, MSC MARC with MANTAT®, which has its own module for high temperature forming analysis. Since the hemisphere configure has its own symmetry plane, half of full configuration was taken account for analysis. The initial blank was discretized by 4 node isoparametric shell elements with the nominal thickness of $7.62 \mathrm{~mm}$ with pre-profiled thickness variation. Based on the analysis, the gas pressure profile for forming process was obtained to maintain the superplastic strain rate condition. The input data is presented in Table I. The maximum pressure was limited to $5 \mathrm{MPa}$ to prevent a possible leaking in the tool.

TABLE I: INPUT DATA FOR FEM ANALYSIS

\begin{tabular}{|l|l|}
\hline \multicolumn{1}{|c|}{ Item } & \multicolumn{1}{|c|}{ Value } \\
\hline Flow stress equation, MPa & $\sigma=K_{\epsilon} \cdot m, \mathrm{~K}=456, \mathrm{~m}=0.44$ \\
\hline Friction factor $(\mu)$ & 0.1 \\
\hline Friction model (Shear friction) & $\sigma_{t} \leq \mu\left(\frac{Y}{\sqrt{3}}\right)^{2} \operatorname{atan}\left(\frac{v_{r}}{\mathrm{C}}\right), \mathrm{C}=1.0$ \\
\hline Min. pressure, MPa & 0.00001 \\
\hline Max. pressure, MPa & 5.0 \\
\hline Optimal strain rate & $4 \times 10^{-4} / \mathrm{s}$ \\
\hline
\end{tabular}

In biaxial bulging tests, the strain rate and the strain rate sensitivity were obtained [5], and the forming temperature of $460{ }^{\circ} \mathrm{C}$ was selected in this study. Since the process of superplastic forming is performed at elevated temperature, a high alloy steel tool was designed to permit argon gas pressurization at the center of the article and installed in a superplastic forming press as shown in Fig. 1 [6]. The press has two metallic platens and in the loading position they will be in parallel horizontal planes, one above the other and, in between, forming tool will be positioned. The lower platen is installed on a moving rail for easy tool change and part loading. The tool with hemispherical cavity of $350 \mathrm{~mm}$ in diameter is capable of applying the back pressure to suppress cavitation and simultaneously maintaining the sealing condition at the forming temperature and high pressure.

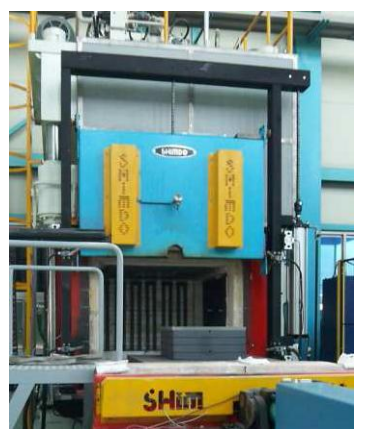

(a)

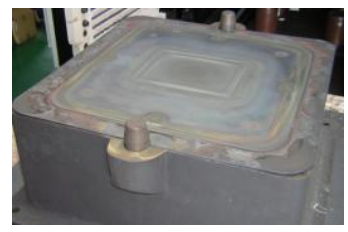

(b)
Fig. 1. (a) Superplastic forming press [6], and (b) a forming tool.

The computer based automatic control system was developed to accurately control gas pressure [6]. Main control valve is Toko-Valex ${ }^{\circledR}$ flow control valve with diaphragm type integral positioner. The accuracy of the pressure regulation at $5 \mathrm{MPa}$ is within $0.5 \%$. The heating system is supplied with a step-down transformer to assure 380 volt line to line maximum at the $\mathrm{SiC}$ heating elements. At $50 \%$ of maximum loading temperature, the electric power consumed is expected about $250 \mathrm{~kW}$. The heated zones are divided to 4 zones and each heated zone is monitored and controlled with programmable logic controller. The heating chamber is insulated with refractory blocks and water cooling is forced in the upper bolster plate to protect press frame structure from heating.

\section{RESULTS AND DISCUSSION}

Microstructure of the specimen is shown in Fig. 2. It is shown that some precipitates are found at the grain boundaries, since the high dislocation density is suitable for the diffusion of the solute elements at the grain boundaries. It is notable that the phase $\# 3\left(\mathrm{Al}_{3} \mathrm{Zr}\right)$ in Fig. $1(\mathrm{~b})$ is important to maintain stable microstructure during superplastic forming process at the elevated temperature. The melting temperature of this phase is $1,350^{\circ} \mathrm{C}$ and it prevents the grain boundary from migrating so to maintain the grain size constant during superplastic forming process. This phase is known to inhibit recrystallization of aluminum alloy at elevated temperature [7].

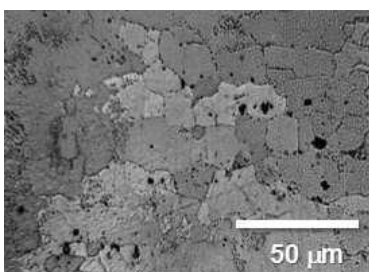

(a)

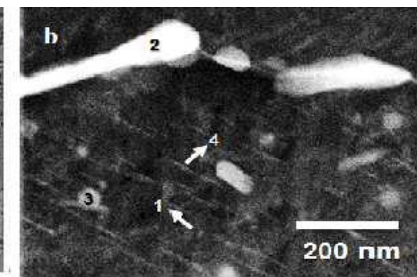

(b)
Fig. 2. Microstructure from (a) metallurgical microscope, and (b) transmission electron microscope showing precipitates.

The gas pressure profile obtained from the analysis was employed in the superplastic forming process as shown in Fig. 3(a) and compared with actual pressure measured. It is interesting to note that the low gas pressure of about $1 \mathrm{MPa}$ was applied until the 2,900 sec, and then the pressure was increased to $5 \mathrm{Mpa}$. This is because initially it is the free bulging without any friction. When circumferential area begins to contact the tool, the material movement is relatively limited and the material in contact with the tool deform plastically. Therefore, it needs higher pressure for further forming. From the analysis, the hemisphere is expected to be completed in 3,420 sec as shown in Fig. 4(c). The thickness profile in Fig. 3(b) indicates the thickness of the final hemisphere shows thinner over the entire area that that from the analysis. This may be due to the fact that the friction between the material and the tool surface is higher than that from the analysis, so that the material is not sliding as expected. This difference can be adjusted by applying appropriate friction coefficient and remodeling the radius of curvature of tool entry contact region so to increase the material movement. In addition to this, the heating time for this article is much longer than that for small biaxial bulging test. Therefore, the actual strain rate sensitivity for forming the hemisphere of $350 \mathrm{~mm}$ in diameter might be lower than that measured from the small bulging test specimen of 100 $\mathrm{mm}$ in diameter since the former underwent the longer exposure to the forming temperature due to the heavier capacity of the tool. The thickness profile from the analysis was not continuous because the specimen had the stepwise initial thickness variation. The general trend of thickness 
variation is acceptable and further study to predict precise profile is under investigation in order to reduce the difference.

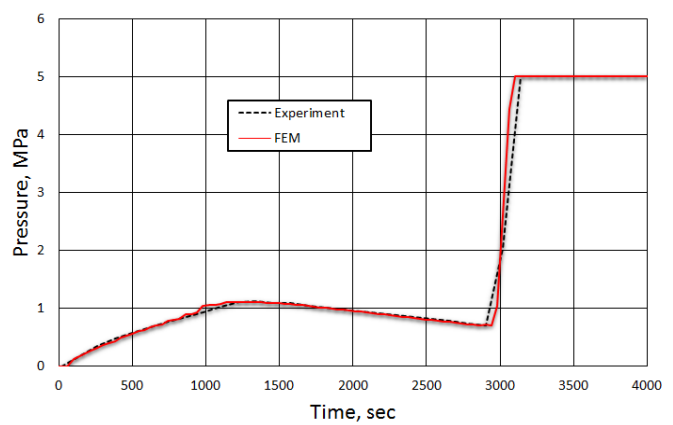

(a)

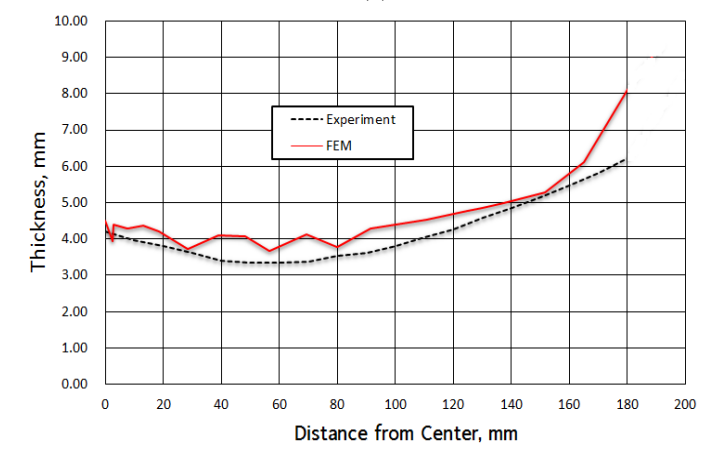

(b)

Fig. 3. (a) Comparison of experimental and analytical results, (a) gas pressurization profile, and (b) thickness profile.

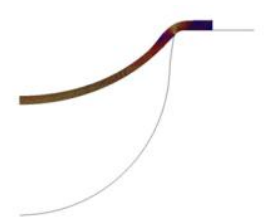

(a)

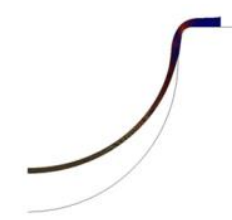

(b)

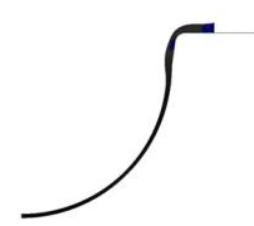

(c)
Fig. 4. Result of hemispherical forming from finite element analysis after (a) $1,020 \mathrm{sec}$, (b) $2,220 \mathrm{sec}$, and (c) 3,420 sec.

The schematic drawing of the tool is shown in Fig. 5. Superplastic forming tools must be durable at operating temperatures during continuous forming process and especially dimensional stability and corrosion resistant property are required. The heat resistant alloy with cobalt and tungsten (28Cr-48Ni-5W-3Co) was chosen for the tool.

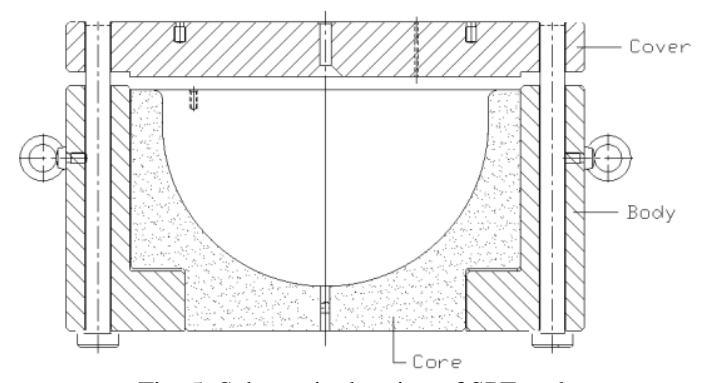

Fig. 5. Schematic drawing of SPF tool.

Fig. 6 shows photographs of of a hemispherical dome of $350 \mathrm{~mm}$ in diameter formed by superplastic forming. The geometry was confirmed with spherical template as shown in Fig. 6 (b). The hemispherical dome has a cylindrical region of $40 \mathrm{~mm}$ at periphery to be joined with other component. This cylindrical area increases friction between the material and the tool surface so to produce the difference in Fig. 3(b). At the edge of the hemispherical dome, there is a constraint around the periphery, leading to a plane-strain stress state. Generally the stress gradient in a forming dome causes a more thinning at the pole and continuously decreases toward to periphery [8], [9]. However, in this study relatively uniform thickness profile was obtained and the thickness at the center was not the minimum. The minimum thickness was obtained at the location about $45 \mathrm{~mm}$ from the center as shown in Fig. 3(b). This demonstrates the current method can decrease the thickness variation of superplastic hemispherical dome in practical application.

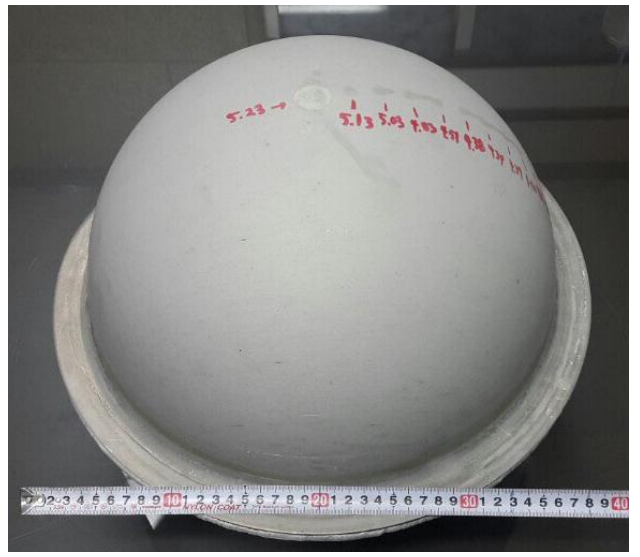

(a)

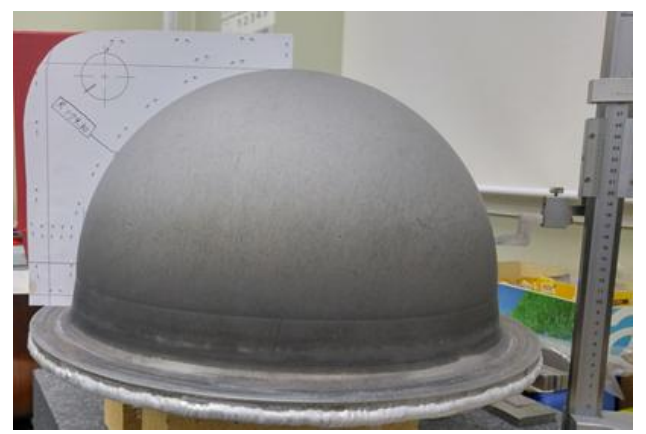

(b)

Fig. 6. Photographs of a hemispherical dome formed by superplastic forming (a) for thickness measurement, and (b) for spherical and height measurement.

\section{CONCLUSION}

Aluminum-lithium alloys due to their low densities, high elastic modulus, and tendency to superplastic forming have been regarded as important structural materials in aerospace industry. Aluminum-lithium 2195 alloy has replaced AA2219 which was being conventionally used for the external tank of the space shuttles.

In this study, superplastic forming of a hemispherical dome is demonstrated with AA2195. Finite element simulation has been carried out using commercial finite element code to predict the superplastic forming profile and the forming temperature was $460{ }^{\circ} \mathrm{C}$ to form a complete hemispherical dome with diameter of $350 \mathrm{~mm}$ for $3,420 \mathrm{sec}$. The programmed gas pressure was applied and the maximum pressure was limited to $5 \mathrm{MPa}$ for practical reason. The distribution of thickness within the hemisphere was investigated and confirmed the analytical result. In this study 
the thickness at the pole of the hemisphere was not the minimum, which indicates uniform thickness distribution is possible by using pre-profile thickness. The process development for manufacturing a hemispherical dome with aluminum-lithium 2195 alloy has been successfully achieved with superplastic forming technology. This process is industrially important to manufacture aerospace components like reaction control tanks, pressure bottles and other parts with complex configuration with aluminum lithium alloy 2195.

\section{ACKNOWLEDGMENT}

This study was supported by the Korean Ministry of Science, ICT and Future Planning (MSIP) through KSLV-II Program.

\section{REFERENCES}

[1] A. K. Mukherjee, J. E. Bird, and J. E. Dorn, "Experimental correlations for high temperature creep," ASM Trans. Q., vol. 62, pp. 155-215, 1969.

[2] J. E. Bird, A. K. Mukherjee, and J. E. Dorn, "Correlations between High-Temperature Creep Behavior and Structure," in Proc. Symposium on Quantitative Relation Between Properties and Microstructure, Haifa, Israel, 27 July-1 August, 1969, pp. 225-342.

[3] H. S. Lee, J. H. Yoon, and J. T. Yoo, "Superplastic blow forming of steel and titanium alloys for aerospace parts," TMS2010, The Mineral, Metals \& Materials Society, Seattle, WA, USA, vol. 3, pp. 253-260, 14-18 February, 2010.

[4] H. M. Flower, G. J. Boyle, R. J. Dashwood, and R. Grimes, "Influence of zirconium precipitation on the superplastic behavour of the Al-6Cu-Zr alloy AA2004," in Proc. 8th International Conference on Superplasticity in Advanced Materials, pp. 389-394, 2004.

[5] J. H. Yoon and H. S. Lee, "Material characterization of duplex stainless steel by superplastic free bulging test," Materialwissenschaft und Werkstofftechnik, vol. 43, pp. 805-809, 2012.

[6] H. S. Lee, J. H. Yoon, and J. T. Yoo, "Development of hot press machine for superplastic forming technology," Advanced Materials Research, vol. 228-229, pp. 563-567, 2011.
[7] H. Adachi, K. Osamura, K. Kikuchi, and J. Kusiu, "Effect of Zr addition on dynamic recrystallization during hot extrusion in $\mathrm{Al}$ alloys," Materials Transactions, vol. 46, pp. 211-214, 2005.

[8] A. Dutta and A. K. Mukherjee, "Superplastic forming: an analytical approach," Materials Science and Engineering, A157, pp. 9-13, 1992

[9] X. D. Ding, H. M. Zbib, C. H. Hamilton, and A. E. Bayoumi, "On the optimization of superplastic blow forming processes," Journal of Manufacturing Science and Performance, vol. 4, pp. 474-485, 1995.

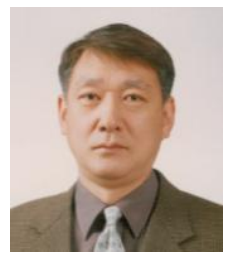

Ho-Sung Lee is a professor at University of Science and Technology and a principal researcher at Korea Aerospace Research Institute. He received his B.S. in Materials Engineering from Hanyang University and attended the graduate school at University of North Carolina at Chapel Hill. His Ph.D. in Materials Science and Engineering is from University of California-Davis (1990). Since then, he has been a principal researcher at KARI, Korea Aerospace Research Institute, which is a national institute. His research interest is the lightweight aerospace materials and manufacturing for space launcher and spacecraft, including superplastic forming, diffusion bonding, friction stir welding, Al-Li alloys, titanium alloys and composite materials.

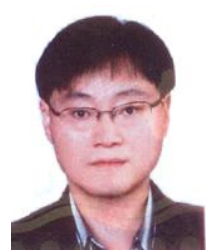

Jong-Hoon Yoon is a principal researcher at Korea Aerospace Research Institute. He attended Korea Advanced Institute of Science and Technology, where he received his Doctor's degree in Mechanical Engineering in 1998. Since then, he is actively working on structure and manufacturing of space launcher vehicle.

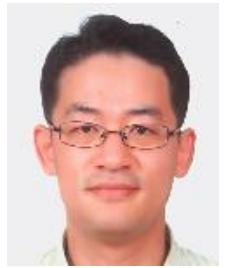

Joon-Tae Yoo is a senior researcher at Korea Aerospace Research Institute. He attended Korea Advanced Institute of Science and Technology, where he received his Doctor's degree in Mechanical Engineering in 2013. He is actively working on structure and welding of tanks for space launcher vehicle. 